\title{
MICRO-COMPUTED TOMOGRAPHIC EVALUATION OF OSSEOINTEGRATION OF TRABECULAR DENTAL IMPLANTS IN A RABBIT MODEL
}

\author{
Hesham Marei* and Haytham Al-Mahalawy**
}

\begin{abstract}
Purpose: To evaluate the osseointegration at the coronal and apical threaded parts of Trabecular Metal (TM) dental implants using micro-CT and comparing it to Tapered Screw Vent (TSV) dental implants.
\end{abstract}

Materials and methods: Ten rabbits were included in this study. Each rabbit received one TM implant $(3.7 \times 10 \mathrm{~mm})$ inserted into the right distal femoral condyle as a test group, and one TSV implant of the same length and diameter inserted into the left distal femoral condyle as a control. Two parts from each implant, the apical and coronal threaded parts to the porous tantalum trabecular material, were selected for the analysis using micro CT.

Results: There were significantly higher mean values for the amount of bone implant contact and bone volume at the apical part of the TM implants when compared with the TSV implants, while there was no significant difference at the coronal part.

Conclusion: The presence of the porous tantalum trabecular material more toward the apical part have created a favourable osteoconductive environment that has reflected on a higher amount of bone implant contact and bone volume at the apical threaded part of the TM implants than that of the equivalent part of the TSV implants.

KEY WORDS: Osseointegration, tantalum, trabecular metal implants, screw vent implants, micro-computed tomography.

\section{INTRODUCTION}

The goal of the current development in implant design and materials is to improve the success rate of dental implants in sites with poor bone quality, and to provide more predictable outcomes for immediately loaded dental implants. The success of dental implants depends on achieving and maintaining "a direct structural and functional connection between ordered living bone and the surface of load-carrying dental implants" in a process defined as osseointegration. ${ }^{1}$ In attempts to improve osseointegration, various surface modifications methods such as grit-blasting, acidetching, surface coating have been suggested..$^{2-4}$

* Oral and Maxillofacial Surgery Department, Faculty of Dentistry, Suez Canal University, Ismailia, Egypt.

** Oral and Maxillofacial Surgery Department, Faculty of Dentistry, Fayoum University, Fayoum, Egypt. 
Implants with porous surface coating have been theorised to enhance osseointegration because bone ingrowth into the surface pores provides a mechanical interlocking at the bone-implant interface ${ }^{5-7}$ Multiple studies have shown that the amount of achievable bone ingrowth is affected by the pore size, shape, and the amount and connectivity of the porosity thickness. ${ }^{8-10}$ Large size pores (more than $300 \mu \mathrm{m}$ ) enhances osteon development and support more vascularized bone ingrowth inside a porous material.,11 However, the amount of bone ingrowth is limited by the presence of porosity on the surface coating only.

Recently, a highly porous tantalum trabecular material (PTTM) with up to $80 \%$ porosity, and a structure and function comparable to cancellous bone was used in hip and knee reconstructive surgery. ${ }^{12,13}$ The same material was used in the fabrication of dental implants known as Trabecular Metal (TM) dental implants (Trabecular Metal Dental Implant, Zimmer Dental Inc., Carlsbad, CA, USA). It is formed of a titanium alloy with a tantalum midsection to achieve bone anchorage through osseoincorporation, a combination of osseointegration (bone ongrowth) and bone ingrowth into the tantalum section. ${ }^{14}$

Previous experimental studies of canine mandible and hip models showed a combination of osseointegration and bone ingrowth inside the porous tantalum sections. ${ }^{15,16}$ The depth of such bone ingrowth varied from $0.2 \mathrm{~mm}$ to the maximal limit of $2 \mathrm{~mm} .{ }^{15}$ The same results were attained via the histologic analysis of implants placed in fresh extraction sockets, as there was bone ingrowth into the porous tantalum structure. However, such bone ingrowth did not result in significant differences in mean implant stability quotients (ISQ), when implants were inserted in either fresh extraction sockets ${ }^{17}$ or healed bony sites at $0,2,4,8$, and 12 weeks of implant placement. ${ }^{16}$

Another in-vitro study showed that tapered screw vent (TSV) implants (Tapered Screw Vent Dental Implant, Zimmer Dental Inc., Carlsbad, CA, USA) have a higher stability in soft bone than TM implants, while in dense bone there was no significant difference between the 2 types of implants. ${ }^{18}$ The authors attributed such differences to the presence of threads along the entire surface of TSV implants compared to the TM implants, which have threads only at the coronal and apical portions. The authors called for more studies to "understand how the primary stability of this new implant design might be improved for immediate loading protocols".

Different studies reported that achieving primary stability in soft bone requires choosing implant design that allows for increasing the surface of bone to implant contact, ${ }^{19}$ the presence of threads in a tapered-design implant, ${ }^{20,21}$ and the use of selftapping implants as this can optimise the stability through lateral bone condensation. ${ }^{22}$

Although there were previous studies that measured and proved histologically the bone ingrowth inside the tantalum midsection of TM implants, it is still important to determine how the bone heals around the coronal and apical parts (threaded part) of TM implants, which are the major interlocking areas to achieve primary stability. This might provide an understanding of the ideal location of the threaded part in relation to the tantalum part along the whole implant length to achieve improved primary stability and better osseointegration. Therefore, the aim of this study was to evaluate the osseointegration at the coronal and apical parts of TM implants using micro-CT by comparing it to TSV implants. The independent variable is the implant design, while the outcome variables are the amount of bone implant contact and bone volume at $500 \mu \mathrm{m}$ around the threaded part in both the TM and TSV implants. Our hypothesis is that there should be no significant difference between both designs at the threaded part. 


\section{MATERIALS AND METHODS}

\section{Study design}

The study included ten adult male $(\mathrm{N}=10)$ white New Zealand rabbits (6-9 months age and 3.5-4.5 $\mathrm{kg}$ ). The sample size was calculated based on mean bone implant contact for test and control groups (35 and 25) and expected standard deviation of 7, which was recorded in previous similar study. ${ }^{17} \mathrm{~A}$ minimum sample size of 8 implants per group is needed to obtain a power of $80 \%$ with a confidence level of $95 \%$.

Each rabbit received one TM implant inserted into the right distal femoral condyle as a part of the test group, and one TSV implant inserted into the left distal femoral condyle that served as a control.

\section{Surgical procedure}

The same surgeon performed all the surgical procedures. A $2 \mathrm{~cm}$ skin incision was performed on the lateral surface of the hind leg. The lateral bone surface of the distal condyle was exposed after reflection of the periosteum. The site for implant insertion was at the middle of the head of the femur. The implant site was prepared according to the manufacture's instructions using a low rotational drill speed (800 rpm) under constant irrigation with sterile saline.

Ten TM dental implants $(3.7 * 10)$ were inserted into the right distal femoral condyle, while $10 \mathrm{TSV}$ dental implants of the same length and diameter were inserted into the left distal femoral condyle. All the implants were submerged to the bone level, and cover screws were inserted (Figure 1). The wound was closed using Vicryl 3-0 resorbable sutures. Postoperative injections of non-steroidal antiinflammatory $(1.5 \mathrm{mg} / \mathrm{kg}$ of diclofenac sodium) and antibiotics $(15 \mathrm{mg} / \mathrm{kg}$ of oxytetracycline) were administered for 3 days. All the rabbits were sacrificed 4 weeks after implant insertion by using an overdose of pentobarbital (Narcoren, Merial GmbH, Hallbergmoos, Germany). The bone specimens contain-



Fig. (1) Surgical insertion of implant at the distal femoral condyle.

ing the implants were preserved in $10 \%$ formaldehyde for micro-computed tomography analysis.

\section{Micro-computed tomography analysis}

Bone specimens containing the implants were scanned using the SkyScan 1172 desktop X-ray micro-CT system (SkyScan, Kontich, Belgium). The SkyScan 1172 system was operated using a highresolution scan with an image pixel size equal to $27.45 \mu \mathrm{m}$, a source voltage of $100 \mathrm{kV}$, an intensity of $100 \mu \mathrm{A}$, and an exposure time of $1500 \mathrm{~ms}$. Scanning was completed by $360^{\circ}$ rotation around the vertical axis, with a rotation step of $0.700^{\circ}$, using a $0.5 \times 0.5$ $\mathrm{mm}$ aluminium cupper filter, and the total scanning time was approximately 1 hour and 15 minutes. We set 4 pixel-size dilations from the implant surface (27.45 $\mu \mathrm{m}$ per dilation) in order to reduce the effect of metal artefact during the measurement of bone morphometric parameters (bone-implant contact $\mathrm{BIC} \%$ and $\mathrm{BV} / \mathrm{TV})$.

For the TM implants, each implant was divided into 3 parts as follows: the apical part (apical to the PTTM part), the PTTM part, and the coronal part (coronal to the PTTM without reaching the bone cortex). The number of slices from the last crosssection showing the most apical end of the implant to the first apical slice of the tantalum part was 75 $(75 \times 27.45=2,058.75 \mu \mathrm{m}=2.06 \mathrm{~mm})$. The number 
of slices for the whole PTTM part was $102(102 \mathrm{x}$ $27.45=2,799.9 \mu \mathrm{m}=2.8 \mathrm{~mm})$, which represents $28 \%$ of the total implant length. The number of slices for the coronal part was $150(150 \times 27.45=$ $4,117.5 \mu \mathrm{m}=4.12 \mathrm{~mm}$ ) (Figure 2).

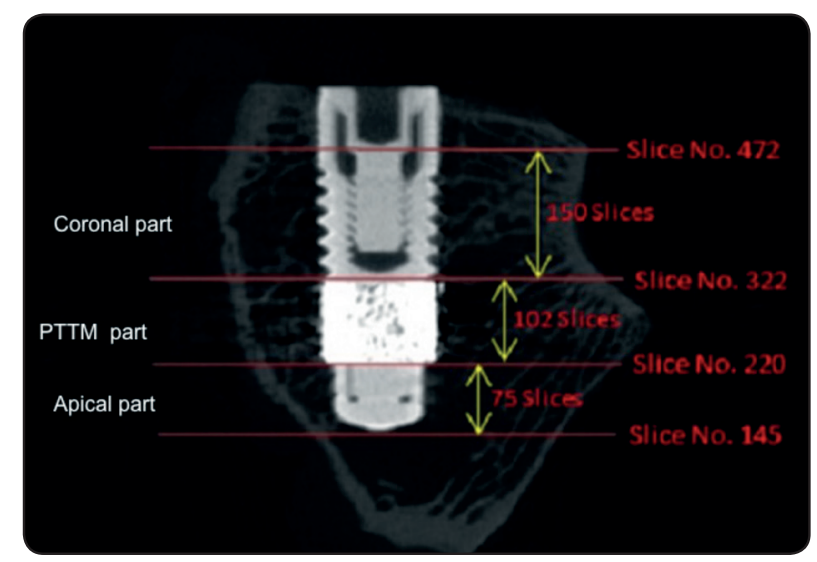

Fig. (2) Micro-CT 2D coronal section of the TM implant showing the division of the implant into 3 parts; apical, porous tantalum trabecular material (PTTM) and coronal.
Two parts from each implant, the apical and coronal parts, were selected for the analysis. The PTTM part was excluded from the analysis due to the excessive generation of metallic artefacts, which might have contaminated the results (Figure 3). For the TSV implants, the part that corresponded to the PTTM in location and size along the TSV implant (102 slices) was excluded from the analysis for standardisation of measurements (Figure 4).

Three bone morphometric parameters were analysed for the apical and coronal parts; the first parameter was defined as a ring with a 1-pixel thickness of $27.45 \mu \mathrm{m}$ following 4 pixel size dilations away from the implant surface. The bone volume to tissue volume (BV/TV) in this ring was considered as the 3D $\mathrm{BIC} \%$. The second parameter was $\mathrm{BV} / \mathrm{TV}$ at $500 \mu \mathrm{m}$, which was defined as a ring that extended $500 \mu \mathrm{m}$ from the implant surface after 4 pixel size dilations. The third parameter was the 2D BIC\%, also after 4 pixel-size dilations, obtained automatically from the software.

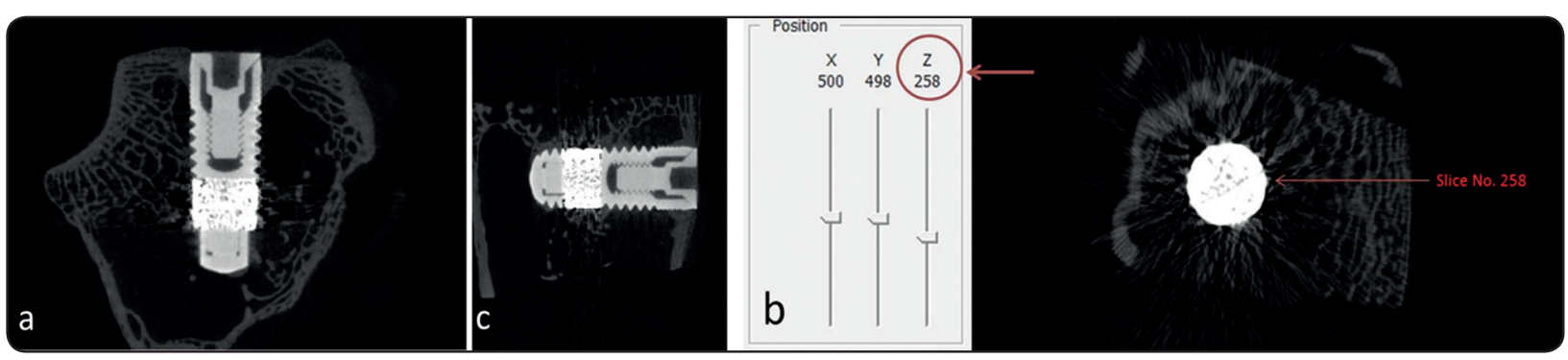

Fig. (3) Micro-CT 2D images of the trabecular implant. (a) Coronal section, showing the artifact concentrated at the PTTM part. (b) Axial section of one slice at the middle of the PTTM part, showing the excessive metallic artefacts radiating from the implant. (c) Sagittal section showing the same findings of the coronal and axial sections.

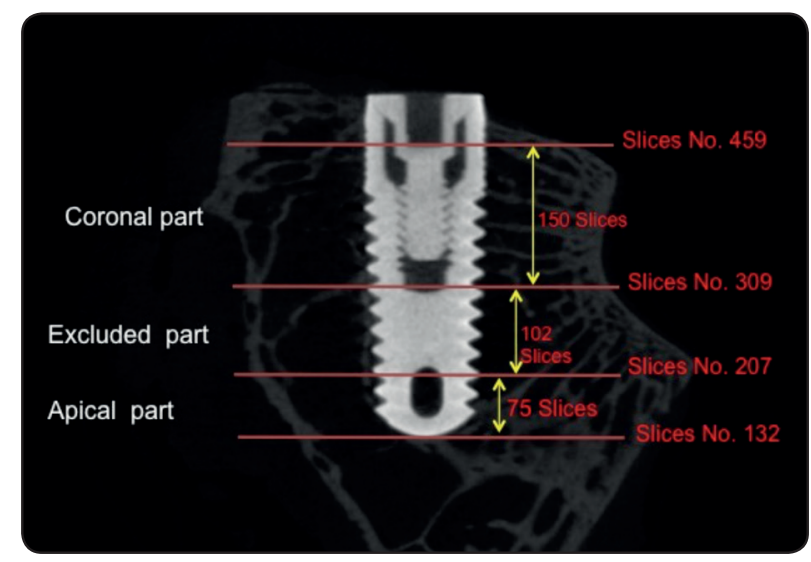

Fig. (4) Micro-CT 2D coronal section of the TSV implant showing the division of the implant into 3 parts; apical, excluded and coronal. 


\section{Statistical analysis}

Statistical data analysis was performed using SPSS version 20 (SPSS Inc., Chicago, IL, USA) software. $P$ value $<5 \%$ is considered as statistically significant. The study outcome variables were tested for the assumption of homogeneity of variance using Levene's test, which proved to be insignificant. Study outcomes were shown to be normally distributed (Skewness was within the range \pm 2 ). Parametric analysis (t-test) was used to calculate differences between the outcomes for the two-implant designs (TM and TSV). Furthermore, the differences between the outcomes at the coronal and apical part for the whole implants were compared. The Pearson correlation was applied to find the correlation between the $2 \mathrm{D}$ and $3 \mathrm{D}$ mean values.

\section{RESULTS}

Eight rabbits survived the surgical procedures uneventfully. Two rabbits died 4 and 5 days postoperatively. For the 8 animals, there were no postoperative complications. At the time of sacrifice, the 16 surgical sites were completely healed, and were available for evaluation.

For the apical part, there was a significant difference for the 3D BIC\% between the TM implants and the TSV implants $(P=0.009)$, as the mean 3D BIC\% $(55.8 \pm 10.6)$ of the TM implants was higher than that of the TSV implants (40.02 \pm 10.0 ). The same significant results were seen for $3 \mathrm{D} \mathrm{BV} / \mathrm{TV}$ and $2 \mathrm{D} \mathrm{BIC} \%(P=0.03$ and 0.02 , respectively), as the mean $3 \mathrm{D} \mathrm{BV} / \mathrm{TV}$ and $2 \mathrm{D} \mathrm{BIC} \%$ (31.4 \pm 11.3 and $64.9 \pm 8.8$, respectively) of the TM implants were higher than that $(19.7 \pm 7.8$ and 53.4 \pm 9.4 , respectively) of the TSV implants.

For the coronal part, there was no significant $(P=0.07)$ difference between the 3D BIC\% of the TM implants $(39.0 \pm 10.3)$ and the TSV implants $(30.3 \pm 7.5)$. The same pattern of results was seen for 3D BV/TV $(P=0.18)$ and 2D BIC\% $(P=0.06)$, as there were no significant differences (Figures 5 and 6) (Table 1).

There was a strong positive correlation between the mean $2 \mathrm{D}(2 \mathrm{D} \mathrm{BIC} \%)$ values and the mean $3 \mathrm{D}$ values (3D $\mathrm{BIC} \%$ and $3 \mathrm{D} \mathrm{BV} / \mathrm{TV}$ ) of the apical part as the Pearson correlation, $\mathrm{r}=0.98$ and 0.84 , respectively. The same strong positive correlation was observed between the mean 2D (2D BIC\%) and $3 \mathrm{D}$ values $(3 \mathrm{D} \mathrm{BIC} \%$ and $3 \mathrm{D} \mathrm{BV} / \mathrm{TV}$ ) of the coronal part ( $r=0.98$ and 0.86 , respectively).

There was a significant difference in the 3D $\mathrm{BIC} \%$ between the apical part and the coronal part for the whole samples $(P=0.003)$, as the mean $3 \mathrm{D}$ BIC\% of the apical part $(47.9 \pm 12.8)$ was higher than that of the coronal part $(34.6 \pm 9.8)$. The same significant results were seen for 3D $\mathrm{BV} / \mathrm{TV}$ and 2D BIC\% ( $P=0.003$ and 0.002 , respectively), as the mean 3D BV/TV and 2D BIC\% $(25.6 \pm 11.1$ and $59.2 \pm 10.6$, respectively) of the apical part were higher than that $(15.5 \pm 8.5$ and $46.5 \pm 10.0$ respectively) of the coronal part (Table 2). 


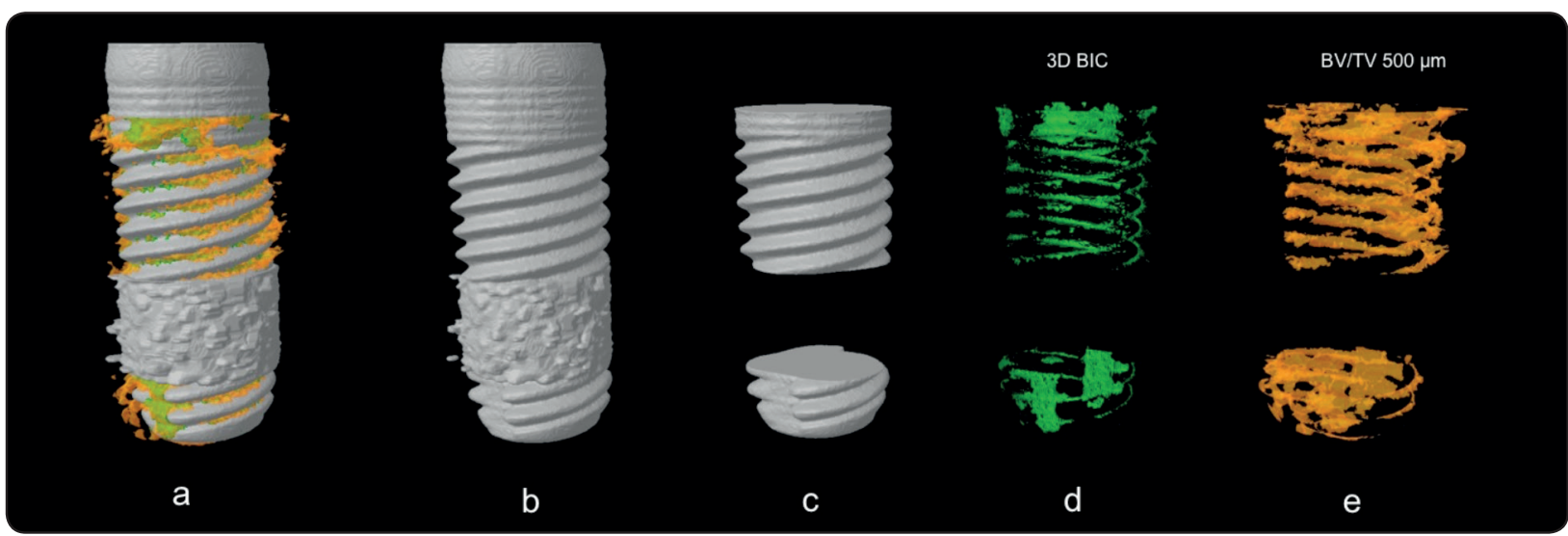

Fig. (5) Micro-CT 3D images of the TM implant. (a) The implant and peri-implant bone in the selected regions of interest. (b) The implant without the bone. (c) The apical and coronal parts after excluding the PTTM part. (d) 3D- bone implant contact. (e) Bone volume / tissue volume at $500 \mu \mathrm{m}$ from the implant surface.

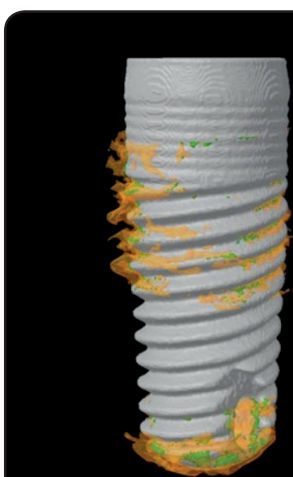

a



b
3D BIC
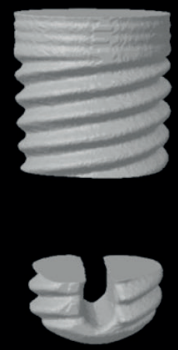

c

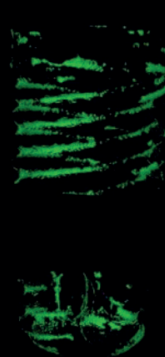

d
BV/TV $500 \mu \mathrm{m}$
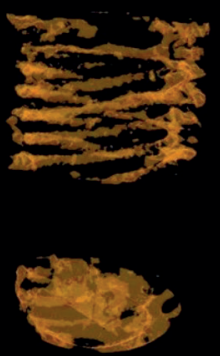

e

Fig. (6) Micro-CT 3D images of the TSV implant. (a) The implant and peri-implant bone in the selected regions of interest. (b) The implant without the bone. (c) The apical and coronal parts. (d) 3D- bone implant contact. (e) Bone volume / tissue volume at $500 \mu \mathrm{m}$ from the implant surface.

TABLE 1: Micro-CT results of the bone morphometric parameters for the TM and TSV implants

\begin{tabular}{|c|c|c|c|c|}
\hline & Group & Mean & Std. Deviation & Pvalue \\
\hline \multirow{2}{*}{ 3D bone implant contact \% (Apical) } & $\mathrm{TM}$ & 55.81 & 10.61 & \multirow{2}{*}{$0.009 *$} \\
\hline & TSV & 40.02 & 10.04 & \\
\hline \multirow{2}{*}{ Bone volume / tissue volume (Apical) } & $\mathrm{TM}$ & 31.45 & 11.30 & \multirow{2}{*}{$0.031^{*}$} \\
\hline & TSV & 19.75 & 7.83 & \\
\hline \multirow{2}{*}{ 2D bone implant contact $\%$ (Apical) } & TM & 64.99 & 8.83 & \multirow{2}{*}{$0.025^{*}$} \\
\hline & TSV & 53.48 & 9.45 & \\
\hline \multirow{2}{*}{ 3D bone implant contact $\%$ (Coronal) } & $\mathrm{TM}$ & 39.00 & 10.37 & \multirow{2}{*}{0.07} \\
\hline & TSV & 30.33 & 7.59 & \\
\hline \multirow{2}{*}{$\begin{array}{l}\text { Bone volume / tissue volume - Top } \\
\text { (Coronal) }\end{array}$} & $\mathrm{TM}$ & 18.40 & 10.34 & \multirow{2}{*}{0.18} \\
\hline & TSV & 12.65 & 5.57 & \\
\hline \multirow{2}{*}{ 2D bone implant contact Top (Coronal) } & TM & 51.12 & 10.54 & \multirow{2}{*}{0.07} \\
\hline & TSV & 42.0188 & 7.68 & \\
\hline
\end{tabular}

*Statistically significant at $P<0.05$. 
TABLE (2) Mean and standard deviation of the bone morphometric parameters at the apical and coronal parts.

\begin{tabular}{|l|c|c|c|c|}
\hline & $\mathrm{N}$ & Mean & Std. Deviation & P value \\
\cline { 1 - 4 } 3D bone implant contact \% (Apical) & 16 & 47.92 & 12.88 & $0.003 *$ \\
\hline 3D bone implant contact \% (Coronal) & 16 & 34.66 & 9.86 & \multirow{2}{*}{$0.003^{*}$} \\
\hline Bone volume / tissue volume (Apical) & 16 & 25.60 & 11.16 & 8.55 \\
\hline Bone volume / tissue volume (Coronal) & 16 & 15.53 & 10.65 & \multirow{2}{*}{$0.002 *$} \\
\hline 2D bone implant contact \% (Apical) & 16 & 59.24 & 10.07 & \\
\hline 2D bone implant contact \% (Coronal) & 16 & 46.57 & \\
\hline
\end{tabular}

*Statistically significant at $P<0.05$.

\section{DISCUSSION}

The aim of this study was to evaluate osseointegration at the coronal and apical parts of TM implants using micro-CT by comparing it to TSV implants to determine how the primary and secondary stability of TM implants can be improved in poor bone quality. The study involved the use of the distal femur of the rabbit as a model for evaluating osseointegration as it was reported in previous studies that the quality of bone in such site is comparable to type IV bone quality of the human posterior maxilla. ${ }^{23,24}$

This study showed significantly higher mean values for the 3D BIC\%, 3D BV/TV, and 2D BIC\% at the apical part when the TM implants were compared with the TSV implants. Such differences could be attributed to two factors. The first is the presence of the PTTM at the lower half of the implant. The PTTM represents nearly $60 \%$ of the lower half of the $3.7 \times 10 \mathrm{~mm}$ implant; thus, it might have created a favourable osteoconductive environment for the osteogenic cells to position and attach on the surface of the implant where they can form bone matrix. Extensive in vitro and in vivo experiments and human studies have disclosed that porous tantalum has good biocompatibility with tissue in growth properties and promoting angiogenesis, making it a potentially improved biomaterial compared to titanium. ${ }^{13,25}$ While titanium allows faster cell proliferation, tantalum enhances the osteoblastic differentiation process. ${ }^{26}$

The second factor is relevant to the difference in the design and shape of both implants at the apical part. The TSV implant involves a vent (hole), while the TM implant involves a solid micro-groove. The outer surface area of a solid micro-groove was higher than that of a hole, which was reflected in a higher amount of $\mathrm{BIC} \%$ and $\mathrm{BV} / \mathrm{TV}$ at the apical part of the TM. The manufacturer claimed that the TM implant provided up to $85.7 \%$ more surface area for osseointegration than TSV implants. ${ }^{27}$ This increase in the surface area is mainly at the lower half of the implant. As at the coronal part, both implants are formed from the same material and follow the same design, which justifies the difference at the apical part only, while there is a lack of difference in the mean values between both implants at the coronal part in the current study. 
Based on a histomorphometric analysis, previous studies reported increased amounts of BIC at the PTTM compared to threaded titanium surfaces. Our study failed to quantify the amount of the 3D BIC\% and 3D BV/TV at the PTTM part using micro-CT due to the high amount of metal artefact. It has been reported in previous studies that tantalum produces more metal artefacts in CT scans than titanium and stainless steel, which made scan interpretation difficult. ${ }^{28,29}$ Metals with high metal atomic mass can produce more artefacts as they produce more attenuation for the X-ray. ${ }^{30}$ The atomic mass of tantalum (180.9 atomic mass units) is 3 times higher than that of titanium (47.8 atomic mass units). However, increasing the exposure parameters (kilovoltage and milliamperage) can reduce the amount of artefacts, but also increases the contrast of the images, making the interpretation of different structures more difficult. ${ }^{31}$ In our study, we used the maximum available parameters (a voltage of $100 \mathrm{kV}$ and an intensity of $100 \mu \mathrm{A}$ ) in the micro-CT machine.

The present study showed significantly higher mean values of 3D $\mathrm{BIC} \%, 3 \mathrm{D} \mathrm{BV} / \mathrm{TV}$, and 2D $\mathrm{BIC} \%$ for the apical than the coronal part. Such differences might be attributed to the difference in blood supply and pressure to different areas in the bone. The blood supply to healthy long bones is derived mainly from the principal nutrient arteries. Nutrient arteries (high-pressure system) enter the long bone cortex and then enter the medullary canal, branching into ascending and descending arteries and supplying the inner $2 / 3$ of the diaphysis via the haversian systems. Furthermore, the perfusion is predominantly centrifugal (that is, blood flow is radiating from inward to outward after delivery to the marrow cavity), while periosteal arteries (low-pressure system) supply the outer $1 / 3$ of the bone, including the cortex..$^{32}$ Therefore, trabecular bone represents a biologically superior tissue if compared to the slowly remodelling cortical bone. ${ }^{33}$ Furthermore, the metabolic activity of osteoblasts depends on a rich blood supply. High blood flow (pressure) allows the continued migration of osteogenic cells to the implant surface, which are then differentiated to the osteoblasts to lay down bone matrix. ${ }^{33}$ Such differences in blood pressure between the bone at the apical and coronal part might have resulted in a greater amount of bone at the apical part.

Our results are partially consistent with Lee et al.'s study, which compared TM and TSV implants in fresh extraction sockets. The authors reported no statistically significant differences in the BIC\% at 2, 4, and 12 weeks between the test and control groups ${ }^{17}$ which is consistent with our results at the coronal part, while Lee et al.'s findings were different from our results at the apical part. This might be attributed to the difference in the area of interest and/or the histological section that was considered during BIC measurement. In the current study, micro-CT analysed the 3D area of interest that was $27.45 \mu \mathrm{m}$ and $500 \mu \mathrm{m}$ from the implant surface, while in the study by Lee et al., the authors histologically analysed a 2D slice of approximately $80 \mu \mathrm{m}$ that was in direct contact with the implant. Furthermore, Lee et al. used 4.1 x 13 mm TM implants. ${ }^{17}$ Such implants have a different proportion in the location and size of the PTTM part compared to the $3.7 \times 10 \mathrm{~mm}$ TM implants used in our study.

The limitation of the current study is that it failed to quantify using micro-CT the amount of BIC and BV/TV at the PTTM part due to a high amount of artefact. Future research should use new micro-CT machines that allow the adjustment of KV, MA, and aluminium filters to reduce the amount of artefact. However, our study also revealed results about the difference in BIC and BV/TV at the apical part of the implants compared to the coronal part, which might lead to industrial implication in future TM implant design and production. The manufacturer might consider moving the PTTM toward the coronal part to improve the osteoconductive environment at 
the coronal part, which has a lower blood supply compared to the apical part. Such modifications will allow non-interruption in the macro-threaded design at the lower half of the implant, possibly contributing to improvement in the initial primary stability.

In conclusion, the presence of the porous tantalum trabecular material more toward the apical part have created a favourable osteoconductive environment that has reflected on a higher amount of bone implant contact and bone volume at the apical threaded part of the TM implants than that of the equivalent part of the TSV implants.

\section{REFERENCES}

1. Adell R, Lekholm U, Rockler B, Brånemark P-I. A 15-year study of osseointegrated implants in the treatment of the edentulous jaw. Int J Oral Surg 1981; 10 (6):387-416.

2. Junker R, Dimakis A, Thoneick M, Jansen JA. Effects of implant surface coatings and composition on bone integration: a systematic review. Clinical oral implants research 2009; 20 Suppl 4:185-206.

3. Wennerberg A, Albrektsson T. On implant surfaces: a review of current knowledge and opinions. The International journal of oral \& maxillofacial implants 2010; 25 (1):6374.

4. Lang NP, Jepsen S. Implant surfaces and design (Working Group 4). Clinical oral implants research 2009; 20 Suppl 4:228-231.

5. do Prado RF, de Vasconcellos LG, de Vasconcellos LM, Cairo CA, Leite Dde O, dos Santos A, et al. In vivo osteogenesis and in vitro Streptococcus mutans adherence: porous-surfaced cylindrical implants vs rough-surfaced threaded implants. The International journal of oral \& maxillofacial implants 2013; 28 (6):1630-1638.

6. Rahmani M, Shimada E, Rokni S, Deporter DA, Adegbembo AO, Valiquette N, et al. Osteotome sinus elevation and simultaneous placement of porous-surfaced dental implants: a morphometric study in rabbits. Clinical oral implants research 2005; 16 (6):692-699.

7. Brentel AS, de Vasconcellos LM, Oliveira MV, Graca ML, de Vasconcellos LG, Cairo CA, et al. Histomorphometric analysis of pure titanium implants with porous surface ver- sus rough surface. Journal of applied oral science : revista FOB 2006; 14 (3):213-218.

8. Otsuki B, Takemoto M, Fujibayashi S, Neo M, Kokubo T, Nakamura T. Pore throat size and connectivity determine bone and tissue ingrowth into porous implants: three-dimensional micro-CT based structural analyses of porous bioactive titanium implants. Biomaterials 2006; 27 (35):5892-5900.

9. Bobyn JD, Pilliar RM, Cameron HU, Weatherly GC. The optimum pore size for the fixation of porous-surfaced metal implants by the ingrowth of bone. Clinical orthopaedics and related research 1980; (150):263-270.

10. Vasconcellos LM, Leite DO, Oliveira FN, Carvalho YR, Cairo CA. Evaluation of bone ingrowth into porous titanium implant: histomorphometric analysis in rabbits. Brazilian oral research 2010; 24 (4):399-405.

11. Karageorgiou V, Kaplan D. Porosity of 3D biomaterial scaffolds and osteogenesis. Biomaterials 2005; 26 (27):54745491.

12. Bobyn JD, Poggie RA, Krygier JJ, Lewallen DG, Hanssen $\mathrm{AD}$, Lewis RJ, et al. Clinical validation of a structural porous tantalum biomaterial for adult reconstruction. The Journal of bone and joint surgery American volume 2004; 86-A Suppl 2:123-129.

13. Patil N, Lee K, Goodman SB. Porous tantalum in hip and knee reconstructive surgery. Journal of biomedical materials research Part B, Applied biomaterials 2009; 89 (1):242251.

14. Bencharit S, Byrd WC, Altarawneh S, Hosseini B, Leong A, Reside G, et al. Development and applications of porous tantalum trabecular metal-enhanced titanium dental implants. Clinical implant dentistry and related research 2014; 16 (6):817-826

15. Bobyn JD, Toh KK, Hacking SA, Tanzer M, Krygier JJ. Tissue response to porous tantalum acetabular cups: a canine model. The Journal of arthroplasty 1999; 14 (3):347-354.

16. Kim DG, Huja SS, Tee BC, Larsen PE, Kennedy KS, Chien $\mathrm{HH}$, et al. Bone ingrowth and initial stability of titanium and porous tantalum dental implants: a pilot canine study. Implant dentistry 2013; 22 (4):399-405.

17. Lee JW, Wen HB, Battula S, Akella R, Collins M, Romanos GE. Outcome after placement of tantalum porous engineered dental implants in fresh extraction sockets: a canine study. The International journal of oral \& maxillofacial implants 2015; 30 (1):134-142. 
18. Romanos GE, Delgado-Ruiz RA, Sacks D, Calvo-Guirado JL. Influence of the implant diameter and bone quality on the primary stability of porous tantalum trabecular metal dental implants: an in vitro biomechanical study. Clinical oral implants research 2016; 00:1-7.

19. Martinez H, Davarpanah M, Missika P, Celletti R, Lazzara R. Optimal implant stabilization in low density bone. Clinical oral implants research 2001; 12 (5):423-432.

20. Mesa F, Munoz R, Noguerol B, de Dios Luna J, Galindo P, O'Valle F. Multivariate study of factors influencing primary dental implant stability. Clinical oral implants research 2008; 19 (2):196-200.

21. Kan JY, Roe P, Rungcharassaeng K. Effects of implant morphology on rotational stability during immediate implant placement in the esthetic zone. The International journal of oral \& maxillofacial implants 2015; 30 (3):667-670.

22. Markovic A, Calvo-Guirado JL, Lazic Z, Gomez-Moreno G, Calasan D, Guardia J, et al. Evaluation of primary stability of self-tapping and non-self-tapping dental implants. A 12-week clinical study. Clinical implant dentistry and related research 2013; 15 (3):341-349.

23. Chowdhary R, Jimbo R, Thomsen CS, Carlsson L, Wennerberg A. The osseointegration stimulatory effect of macrogeometry-modified implants: a study in the rabbit. Clinical oral implants research 2014; 25 (9):1051-1055.

24. Park JW, Jang IS, Suh JY. Bone response to endosseous titanium implants surface-modified by blasting and chemical treatment: a histomorphometric study in the rabbit femur. Journal of biomedical materials research Part B, Applied biomaterials 2008; 84 (2):400-407.

25. Bobyn JD, Stackpool GJ, Hacking SA, Tanzer M, Krygier JJ. Characteristics of bone ingrowth and interface mechanics of a new porous tantalum biomaterial. The Journal of bone and joint surgery British volume 1999; 81 (5):907914.
26. Stiehler M, Lind M, Mygind T, Baatrup A, Dolatshahi-Pirouz A, Li H, et al. Morphology, proliferation, and osteogenic differentiation of mesenchymal stem cells cultured on titanium, tantalum, and chromium surfaces. Journal of biomedical materials research Part A 2008; 86 (2):448-458

27. Zimmer Trabecular Metal Dental Implant. http://www.zimmerbiometdental.com: Zimmer Dental.

28. Silverman PM, Spicer LD, McKinney R, Jr., Feldman DB. Computed tomographic evaluation of surgical clip artifact: tissue phantom and experimental animal assessment. Computerized radiology : official journal of the Computerized Tomography Society 1986; 10 (1):37-40.

29. Weese JL, Rosenthal MS, Gould H. Avoidance of artifacts on computerized tomograms by selection of appropriate surgical clips. American journal of surgery 1984; 147 (5):684-687.

30. Sancho-Puchades M, Hammerle CH, Benic GI. In vitro assessment of artifacts induced by titanium, titanium-zirconium and zirconium dioxide implants in cone-beam computed tomography. Clinical oral implants research 2015; 26 (10):1222-1228.

31. Kataoka ML, Hochman MG, Rodriguez EK, Lin PJ, Kubo $\mathrm{S}$, Raptopolous VD. A review of factors that affect artifact from metallic hardware on multi-row detector computed tomography. Current problems in diagnostic radiology 2010; 39 (4):125-136.

32. Marenzana M, Arnett TR. The Key Role of the Blood Supply to Bone. Bone research 2013; 1 (3):203-215.

33. Davies JE. Understanding peri-implant endosseous healing. Journal of dental education 2003; 67 (8):932-949. 